\title{
Analysis of physico-chemical properties and heavy metals in drinking water from different sources in and around Ranchi, J harkhand, India
}

\author{
Priscilla Kerketta, Sushma Lalita Baxla, Ravuri Halley Gora, Suruchi Kumari and Rustam Kumar Roushan \\ Ranchi Veterinary College, \\ Birsa Agricultural University, Ranchi, J harkhand 834006, India. \\ Corresponding author: Priscilla Kerketta, email: priscillak802@gmail.com \\ Received: 10-11-2012, Accepted: 27-12-2012, Published online: 31-03-2013
}

How to cite this article: Kerketta P, Baxla SL, Gora RH, Kumari S and Roushan RK (2013) Analysis of physico-chemical properties and heavy metals in drinking water from different sources in and around Ranchi, Jharkhand, India, Vet World 6(7): 370-375, doi: 10.5455/vetworld.2013.370-375

\begin{abstract}
Aim: The present study was undertaken to determine the physico-chemical properties and heavy metals in drinking water samples collected from different sources in and around Ranchi, Jharkhand, India.

Materials and Methods: A total number of 100 water samples were collected from different sources like hand pumps (44), wells (27), taps (20), rivers (3) and ponds (6). The gross appearance, taste, odour, temperature, $\mathrm{pH}$, dissolved oxygen, biochemical oxygen demand, alkalinity, conductivity, total dissolved solid, salinity and the concentration of lead and cadmium were analyzed.

Results: Water samples were transparent, odourless and taste was agreeable except rivers and ponds. The mean value of temperature (24.8 \pm 0.416 to $30.7 \pm 0.819), \mathrm{pH}(6.4 \pm 0.1$ to $7.3 \pm 0.1)$, dissolved oxygen $(3.9 \pm 0.05$ to $8.95 \pm 0.15)$, biochemical oxygen demand ( $2.3 \pm 0.7$ to $7.5 \pm 0.409)$, alkalinity ( $35 \pm 1$ to $144.75 \pm 40.359)$, conductivity ( $83 \pm 1.15$ to $1508 \pm 0)$, total dissolved solid (73 \pm 1 to $1422 \pm 0)$ and salinity $(0.016 \pm .014)$. Mean levels $(\mathrm{ppm})$ of lead $(\mathrm{Pb})$ was $(0.01 \pm 0.00$ to $0.11 \pm 0.03)$ and cadmium (Cd) was $(0.04 \pm 0.01$ to $0.11 \pm 0.03)$.

Conclusion: Out of water samples collected from above mentioned sources, samples from ponds and rivers were highly contaminated. The $\mathrm{pH}$ of 6 water samples was more than prescribed level, dissolved oxygen in 11 was samples was below than 5 ppm, biochemical oxygen demand in 19 water samples was above 6 ppm, alkalinity in 14 samples were above 120 ppm, conductivity in 2 water samples were above $1400 \mu \mathrm{S} / \mathrm{cm}$, The levels of lead and cadmium was more than the permissible limits.
\end{abstract}

Keywords: alkalinity, BOD, cadmium, DO, lead, pH, physico-chemical properties, TDS

\section{I ntroduction}

Water of good drinking quality is of basic importance to human physiology and man's continued existence depends on its availability [1]. The provision of potable water to the rural and urban population is necessary to prevent health hazards [2,3].

Ground water is generally considered as a safe source of fresh drinking water [4]. But rapid population growth, increasing living standards in urban areas and industrialization have resulted in greater demand of quality water on one hand, while on the other hand, pollution of water sources is increasing steadily. Therefore the ground water is getting polluted and among which wells are generally considered as the worst type of ground water sources in the term of physico-chemical contamination due to the lack of concrete plinth and surrounding drainage system $[5,6]$. The incidence of ground water pollution is highest in urban areas where large volumes of waste discharged into relatively small areas [7]. There are various factors, which are responsible for ground water contamination such as use of fertilizer in farming [8],

This article is an open access article licensed under the terms of the Creative Commons Attribution License (http://creativecommons. org/licenses/by/2.0) which permits unrestricted use, distribution and reproduction in any medium, provided the work is properly cited. seepage from effluent bearing water body [9]. Once the groundwater is contaminated, its quality cannot be restored by stopping the pollutants from the source. Therefore it becomes imperative to regularly monitor the quality of groundwater and to device various means to protect it [10]. The surface water sources, in general, are not acceptable for drinking purpose as these are often loaded by various organic, inorganic and biological constituents [11,12].

In the recent years, the availability and access to fresh water has become the most critical issue in the world. Freshwater is essential to human health, agriculture, industry and natural ecosystems, but is now running scarce in many regions of the world [13]. The desirable properties of water quality should include adequate amount of dissolved oxygen, relatively low organic content, $\mathrm{pH}$ value near neutrality, moderate temperature, and free from excessive amount of infectious agents, toxic substances and mineral matter [14]. Potable water should be free from disease producing microorganisms and chemical substances that are dangerous to health $[15,16]$. Majority of the rural people do not have access to potable water and therefore, depend on well, stream and river waters for domestic use. Excessive use of limited water resources, disposal of various industrial effluents, human wastes 
into water may release heavy metals, which harm both human and animals health [17]. Continuous exposure to heavy metals to animals and humans cause hepatotoxicity and nephrotoxicity. So, periodical estimation of level of heavy metals in water is necessary.

\section{Materials and Methods}

A total of 100 samples were taken from different sources like hand-pumps (44), wells (27), taps (20), ponds (6) and rivers (3) were collected in and around Ranchi.

Method of sample collection: Water samples were randomly collected from different sources at varying interval in thoroughly washed and sterilized bottle. Physico-chemical analysis was done within 48 hours and the samples were stored at room temperature.

\section{Physico-chemical analysis:}

Gross appearance, odour and taste: The water samples were observed with naked eyes for gross appearance and examined for offensive odour through the subjective organoleptic assessment.

Temperature: The temperature was recorded in Celsius $\left({ }^{0} \mathrm{C}\right)$ with the help of mercury thermometer.

pH: $\mathrm{pH}$ of the water was determined with the help of $\mathrm{pH}$ meter (Model Digital pH meter 335).

Dissolved oxygen (DO): Dissolved oxygen of water was determined as per method [18] and expressed in terms of ppm.

Biochemical oxygen demand (BOD): BOD was determined as per the method [19] and was expressed in terms of ppm. The method involves, measuring difference of oxygen concentration of the sample before and after incubation of 5 days at $20^{\circ} \mathrm{C}$.

Alkalinity: Alkalinity was determined as per the method [20] and expressed in terms of ppm.

Conductivity: The conductivity of water was determined with the help of Conductivity meter (Model Inolab Cond 720) and expressed in terms of $\mu \mathrm{S} / \mathrm{cm}$.

Total dissolved solids (TDS) : The total dissolved solids of water was determined by Conductivity meter (Model Inolab Cond 720) and expressed in mg/l.

Salinity: The salinity content of water was determined by Conductivity meter (Model Inolab Cond 720) \& expressed in terms of ppt.

Heavy metal estimation: The estimation of Lead (pb) and Cadmium $(\mathrm{Cd})$ of the water samples was done as per method [21] by SL-176, Double beam atomic absorption spectrophotometer, ELICO.

\section{Results and Discussion}

The results and discussion of physico-chemical properties of water samples was given below.
Gross appearance, odour and taste: Water samples from most of the sources were transparent, odourless and taste was agreeable except water from rivers and ponds which were highly turbid and foul smelling, which appears aesthetically objectionable for human and animal use.

Temperature: Water samples were collected and analyzed between March to July. The temperature of water ranged from 24 to $31^{\circ} \mathrm{C}$. The mean temperature ranged from $24.8 \pm 0.416$ to $30.7 \pm 0.819{ }^{\circ} \mathrm{C}$ (Table- 1 ), almost similar findings were observed by [22], who examined water samples collected from Anand district of Gujarat and reported that the temperature of water samples ranged between 28 to $31^{\circ} \mathrm{C}$. The high temperature may be due to increase in atmospheric temperature during summer season. In a study conducted by [23], temperature of ranged from 21 to $30^{\circ} \mathrm{C}$ in water of Triveni lake of Amaravati district in India. In the similar study conducted in Bichi Local Government Area of Kano State of Nigeria by [24] and reported the mean temperature ranged from 24.5 to $26.2^{\circ} \mathrm{C}$. The low temperature may be due to winter season.

pH: The overall $\mathrm{pH}$ ranged from 6.3 to 7.4 with the mean values ranged from $6.4 \pm 0.1$ to $7.3 \pm 0.1$ (Table- 1 ). It was found that 6 water samples were below the prescribed level of 6.5 , lowest $\mathrm{pH} 6.3$ was reported which is slightly acidic. These findings were supported by the results of [25], who reported $\mathrm{pH}$ of water samples ranging from 6.8 to 7.3. The $\mathrm{pH}$ of water ranged from 6.8 to 8.3 in a similar study conducted by [26] in the selected area of Ghazipur city, India.

Dissolved oxygen: The dissolved oxygen content of water ranged from 2.6 to $11 \mathrm{ppm}$ and the mean values ranged from $3.9 \pm 0.05$ to $8.95 \pm 0.15 \mathrm{ppm}$ (Table-1). It was observed that in 11 samples, dissolved oxygen was below 5 ppm. Lower dissolved oxygen content was noticed in ponds $(2.6 \mathrm{ppm})$ and river $(2.9 \mathrm{ppm})$. In a similar study conducted by [27] who reported the DO fluctuated from 7.25 to $16 \mathrm{ppm}$. This variation in dissolved oxygen might be due to temperature, photosynthesis, respiration, aeration, organic waste and sediment concentration [28]. The reduced dissolved oxygen may affect the aquatic life. However, there is no report of ill health effects arising directly from a deficiency of dissolved oxygen in potable water or from its complete absence [29].

Biochemical oxygen demand: The biochemical oxygen demand of the water ranged between 1.6 to 9 ppm with the mean varying from $2.3 \pm 0.7$ to $7.5 \pm 0.409$ ppm (Table-1). In 19 water samples, biochemical oxygen demand was above $6 \mathrm{ppm}$ as prescribed by [30]. According to study conducted by [31] who found biochemical oxygen demand ranging from 0.16 to 11.6 $\mathrm{mg} / \mathrm{l}$. Water with BOD $<4 \mathrm{ppm}$ are considered to be clean water, while those with $>10 \mathrm{ppm}$ are considered to be polluted and unsafe [32]. High BOD has 
Table-1. Physico-chemical properties of drinking water samples (Mean \pm SEM)

\begin{tabular}{|c|c|c|c|c|c|c|c|c|c|c|}
\hline No. & Source & $\begin{array}{c}\text { No. of } \\
\text { samples } \\
\text { examined }\end{array}$ & Temp $\left({ }^{\circ} \mathrm{C}\right)$ & $\mathrm{pH}$ & $\begin{array}{l}\text { Dissolved oxygen } \\
\text { (ppm) }\end{array}$ & $\begin{array}{l}\text { Biochemical oxygen } \\
\text { demand (ppm) }\end{array}$ & $\begin{array}{l}\text { nAlkalinity } \\
\text { (ppm) }\end{array}$ & $\begin{array}{l}\text { Conductivity } \\
(\mu \mathrm{S} / \mathrm{cm})\end{array}$ & TDS (mg/l) & $\begin{array}{l}\text { Salinity } \\
\text { (ppt) }\end{array}$ \\
\hline \multirow[t]{3}{*}{1} & Handpump & 5 & $\begin{array}{l}27-28.6 \\
(28 \pm 0.275)\end{array}$ & $\begin{array}{l}6.5-6.8 \\
(6.6 \pm 0.063)\end{array}$ & $\begin{array}{l}5-8 \\
(7 \pm 0.593)\end{array}$ & $\begin{array}{l}5-7.2 \\
(5.7 \pm 0.954)\end{array}$ & $\begin{array}{l}50-172 \\
(102.8 \pm 20.303)\end{array}$ & $\begin{array}{l}176.5-371 \\
(252.3 \pm 35.610)\end{array}$ & $\begin{array}{l}154-201 \\
(176.8 \pm 7.465)\end{array}$ & 0 \\
\hline & Well & 3 & $\begin{array}{l}25.2-26 \\
(25.73 \pm 0.266)\end{array}$ & $\begin{array}{l}6.7-7 \\
(6.9 \pm 0.1)\end{array}$ & $\begin{array}{l}6.1-10 \\
(8.16 \pm 1.131)\end{array}$ & $\begin{array}{l}3.2-8.6 \\
(5.93 \pm 1.559)\end{array}$ & $\begin{array}{l}84-102 \\
(94 \pm 5.291)\end{array}$ & $\begin{array}{l}198-1008 \\
(521.66 \pm 247.568)\end{array}$ & $\begin{array}{l}114-879 \\
(435.33 \pm 229.15)\end{array}$ & $\begin{array}{l}0-0.1 \\
(0.03 \pm 0.033)\end{array}$ \\
\hline & Tap & 2 & $\begin{array}{l}30.4-31 \\
(30.7 \pm 0.3)\end{array}$ & $\begin{array}{l}6.3-6.5 \\
(6.4 \pm 0.1)\end{array}$ & $\begin{array}{l}8.4-10.8 \\
(9.6 \pm 1.2)\end{array}$ & $\begin{array}{l}5.1-5.3 \\
(5.2 \pm 0.2)\end{array}$ & $\begin{array}{l}72-144 \\
(108 \pm 36)\end{array}$ & $\begin{array}{l}666-680 \\
(673 \pm 7)\end{array}$ & $\begin{array}{l}580-593 \\
(586.5 \pm 6.5)\end{array}$ & $\begin{array}{l}0-0.1 \\
(0.05 \pm 0.05)\end{array}$ \\
\hline \multirow[t]{3}{*}{2} & Handpump & 4 & $\begin{array}{l}27-28.2 \\
(27.8 \pm 0.27)\end{array}$ & $\begin{array}{l}6.5-6.8 \\
(6.62 \pm 0.075)\end{array}$ & $\begin{array}{l}7-9 \\
(8.25 \pm 0.434)\end{array}$ & $\begin{array}{l}6.8-8.4 \\
(7.55 \pm 0.409)\end{array}$ & $\begin{array}{l}48-210 \\
(116 \pm 36.193)\end{array}$ & $\begin{array}{l}130-663 \\
(371.25 \pm 130.088)\end{array}$ & $\begin{array}{l}116-176 \\
(144.5 \pm 12.816)\end{array}$ & 0 \\
\hline & Well & 2 & $\begin{array}{l}25-27 \\
(26 \pm 1)\end{array}$ & $\begin{array}{l}6.7-6.9 \\
(6.8 \pm 0.1)\end{array}$ & $\begin{array}{l}8.8-9.1 \\
(8.95 \pm 0.15)\end{array}$ & $\begin{array}{l}4.2-8 \\
(6.1 \pm 0.1)\end{array}$ & $\begin{array}{l}36-56 \\
(46 \pm 10)\end{array}$ & $\begin{array}{l}76-108 \\
(92 \pm 16)\end{array}$ & $\begin{array}{l}67-215 \\
(141 \pm 74)\end{array}$ & 0 \\
\hline & Tap & 1 & 29 & 7.1 & 5 & 7.1 & 56 & 710 & 504 & 0 \\
\hline \multirow[t]{3}{*}{3} & Handpump & 4 & $\begin{array}{l}27-28.2 \\
(27.7 \pm 0.264)\end{array}$ & $\begin{array}{l}6.8-7.2 \\
(6.8 \pm 0.141)\end{array}$ & $\begin{array}{l}5.6-8 \\
(6.65 \pm 0.537)\end{array}$ & $\begin{array}{l}5.6-9 \\
(6.65 \pm 0.788)\end{array}$ & $\begin{array}{l}68-256 \\
(144.7 \pm 40.359)\end{array}$ & $\begin{array}{l}117-1270 \\
(539.25 \pm 254.428)\end{array}$ & $\begin{array}{l}139-1107 \\
(490 \pm 212.06)\end{array}$ & $\begin{array}{l}0-0.4 \\
(0.1 \pm 0.1)\end{array}$ \\
\hline & Well & 3 & $\begin{array}{l}25.2-26.2 \\
(25.8 \pm 0.305)\end{array}$ & $\begin{array}{l}6.7-7 \\
(6.8 \pm 0.088)\end{array}$ & $\begin{array}{l}5.6-6.9 \\
(6.4 \pm 0.417)\end{array}$ & $\begin{array}{l}5-7.6 \\
(6.4 \pm 0.768)\end{array}$ & $\begin{array}{l}22-90 \\
(54 \pm 19.731)\end{array}$ & $\begin{array}{l}90.7-432 \\
(210.5 \pm 110.842)\end{array}$ & $\begin{array}{l}94-790 \\
(333.6 \pm 228.263)\end{array}$ & $\begin{array}{l}0-0.2 \\
(0.06 \pm 0.066)\end{array}$ \\
\hline & Tap & 2 & $\begin{array}{l}29.4-31 \\
(30.2 \pm 0.8)\end{array}$ & $\begin{array}{l}6.3-6.5 \\
(6.4 \pm 0.1)\end{array}$ & $\begin{array}{l}6.2-7 \\
(6.6 \pm 0.4)\end{array}$ & $\begin{array}{l}5-6.4 \\
(5.7 \pm 0.7)\end{array}$ & $\begin{array}{l}46-124 \\
(85 \pm 39)\end{array}$ & $\begin{array}{l}226-1081 \\
(653.5 \pm 427.5)\end{array}$ & $\begin{array}{l}493-941 \\
(717 \pm 224)\end{array}$ & $\begin{array}{l}0-0.3 \\
(0.15 \pm 0.15)\end{array}$ \\
\hline \multirow[t]{4}{*}{4} & Hand pump & 4 & $\begin{array}{l}26.4-29 \\
(27.45 \pm 0.556)\end{array}$ & $\begin{array}{l}6.5-7 \\
(6.7 \pm 0.110)\end{array}$ & $\begin{array}{l}5.6-11 \\
(7.6 \pm 1.245)\end{array}$ & $\begin{array}{l}5-8 \\
(5.9 \pm 0.686)\end{array}$ & $\begin{array}{l}36-110 \\
(64.5 \pm 17.173)\end{array}$ & $\begin{array}{l}145-1224 \\
(497.5 \pm 248.901)\end{array}$ & $\begin{array}{l}127-1067 \\
(382.2 \pm 228.50)\end{array}$ & $\begin{array}{l}0-0.4 \\
(0.1 \pm 0.1)\end{array}$ \\
\hline & Well & 2 & $\begin{array}{l}24.1-25.8 \\
(24.9 \pm 0.85)\end{array}$ & $\begin{array}{l}6.5-6.7 \\
(6.6 \pm 0.1)\end{array}$ & $\begin{array}{l}7.6-7.7 \\
(7.6 \pm 0.05)\end{array}$ & $\begin{array}{l}5.2-6 \\
(5.6 \pm 0.4)\end{array}$ & $\begin{array}{l}50-83 \\
(66.5 \pm 16.5)\end{array}$ & $\begin{array}{l}132-755 \\
(443.5 \pm 311.5)\end{array}$ & $\begin{array}{l}178-656 \\
(417 \pm 239)\end{array}$ & $\begin{array}{l}0-0.1 \\
(0.05 \pm 0.05)\end{array}$ \\
\hline & Tap & 2 & $\begin{array}{l}28-29.6 \\
(28.8 \pm 0.8)\end{array}$ & $\begin{array}{l}6.8-7.2 \\
(7 \pm 0.2)\end{array}$ & $\begin{array}{l}5-8 \\
(6.5 \pm 1.7)\end{array}$ & $\begin{array}{l}5.4-6 \\
(5.7 \pm 0.3)\end{array}$ & $\begin{array}{l}78-84 \\
(81 \pm 3)\end{array}$ & $\begin{array}{l}128-428 \\
(278 \pm 150)\end{array}$ & $\begin{array}{l}217-372 \\
(294.5 \pm 77.5)\end{array}$ & 0 \\
\hline & River & 1 & 31 & 6.8 & 3.4 & 2.3 & 112 & 397 & 190 & 0 \\
\hline \multirow[t]{4}{*}{5} & Hand pump & 2 & $\begin{array}{l}26-27 \\
(26.5 \pm 0.5)\end{array}$ & $\begin{array}{l}7.1-7.2 \\
(7.1 \pm 0.05)\end{array}$ & $\begin{array}{l}5-8.4 \\
(6.7 \pm 1.7)\end{array}$ & $\begin{array}{l}5.2-5.6 \\
(5.4 \pm 0.2)\end{array}$ & $\begin{array}{l}40-80 \\
(60 \pm 20)\end{array}$ & $\begin{array}{l}147.5-191.2 \\
(169.35 \pm 21.85)\end{array}$ & $\begin{array}{l}130-166 \\
(148 \pm 18)\end{array}$ & 0 \\
\hline & Well & 2 & $\begin{array}{l}26-27.4 \\
(26.7 \pm 0.7)\end{array}$ & $\begin{array}{l}6.5-7.2 \\
(6.8 \pm 0.3)\end{array}$ & $\begin{array}{l}6-8 \\
(7 \pm 1)\end{array}$ & $\begin{array}{l}5.3-5.8 \\
(5.5 \pm 0.25)\end{array}$ & $\begin{array}{l}34-81 \\
(57.5 \pm 23.5)\end{array}$ & $\begin{array}{l}119.2-136 \\
(127.6 \pm 8.4)\end{array}$ & $\begin{array}{l}104-201 \\
(152.5 \pm 48.5)\end{array}$ & 0 \\
\hline & Tap & 2 & $\begin{array}{l}27-28.2 \\
(27.6 \pm 0.6)\end{array}$ & $\begin{array}{l}6.5-7.1 \\
(6.8 \pm 0.3)\end{array}$ & $\begin{array}{l}6-8.4 \\
(7.2 \pm 1.2)\end{array}$ & $\begin{array}{l}5-6.4 \\
(5.7 \pm 0.7)\end{array}$ & $\begin{array}{l}34-36 \\
(35 \pm 1)\end{array}$ & $\begin{array}{l}82.6-84.9 \\
(83.75 \pm 1.15)\end{array}$ & $\begin{array}{l}72-74 \\
(73 \pm 1)\end{array}$ & 0 \\
\hline & ponds & 2 & $\begin{array}{l}30-31 \\
(30.5 \pm 0.5)\end{array}$ & $\begin{array}{l}6.8-7 \\
(6.9 \pm 0.1)\end{array}$ & $\begin{array}{l}3.9-4 \\
(3.9 \pm 0.05)\end{array}$ & $\begin{array}{l}1.6-3 \\
(2.3 \pm 0.7)\end{array}$ & $\begin{array}{l}82-114 \\
(98 \pm 16)\end{array}$ & $\begin{array}{l}304-600 \\
(452 \pm 148)\end{array}$ & $\begin{array}{l}265-522 \\
(393.5 \pm 128.5)\end{array}$ & 0 \\
\hline \multirow[t]{3}{*}{6} & Hand pump & 3 & $\begin{array}{l}26.4-29.2 \\
(28.2 \pm 0.90)\end{array}$ & $\begin{array}{l}6.5-7.1 \\
(6.7 \pm 0.2)\end{array}$ & $\begin{array}{l}5-9.4 \\
(7.3 \pm 1.277)\end{array}$ & $\begin{array}{l}5-8 \\
(6.1 \pm 0.953)\end{array}$ & $\begin{array}{l}56-80 \\
(70.66 \pm 7.423)\end{array}$ & $\begin{array}{l}351-505 \\
(420.33 \pm 45.112)\end{array}$ & $\begin{array}{l}294-440 \\
(361.66 \pm 42.482)\end{array}$ & 0 \\
\hline & Well & 1 & 25 & 6.5 & 7.6 & 5.6 & 70 & 164 & 1422 & 0.7 \\
\hline & Tap & 2 & $\begin{array}{l}29.2-30 \\
(29.6 \pm 0.4)\end{array}$ & $\begin{array}{l}6.6-6.8 \\
(6.7 \pm 0.1)\end{array}$ & $\begin{array}{l}5.2-6.4 \\
(5.8 \pm 0.6)\end{array}$ & $\begin{array}{l}5-5.6 \\
(5.3 \pm 0.3)\end{array}$ & $\begin{array}{l}60-184 \\
(122 \pm 62)\end{array}$ & $\begin{array}{l}1054-1117 \\
(1085.5 \pm 31.5)\end{array}$ & $\begin{array}{l}96-971 \\
(533.5 \pm 437.5)\end{array}$ & $\begin{array}{l}0.3-0.4 \\
(0.35 \pm 0.05)\end{array}$ \\
\hline \multirow[t]{3}{*}{7} & Hand pump & 5 & $\begin{array}{l}24.8-28 \\
(26.7 \pm 0.595)\end{array}$ & $\begin{array}{l}6.5-7 \\
(6.5 \pm 0.112)\end{array}$ & $\begin{array}{l}4.8-11 \\
(7.5 \pm 1.223)\end{array}$ & $\begin{array}{l}5-8 \\
(6.1 \pm 0.582)\end{array}$ & $\begin{array}{l}34-80 \\
(61.6 \pm 7.884)\end{array}$ & $\begin{array}{l}243-1636 \\
(616.6 \pm 257.64)\end{array}$ & $\begin{array}{l}205-1311 \\
(473 \pm 211.176)\end{array}$ & $\begin{array}{l}0-0.6 \\
(0.12 \pm 0.12)\end{array}$ \\
\hline & Well & 3 & $\begin{array}{l}26-27.4 \\
(26.6 \pm 0.416)\end{array}$ & $\begin{array}{l}6.4-7.2 \\
(6.8 \pm 0.24)\end{array}$ & $\begin{array}{l}6.4-8 \\
(7 \pm 0.48)\end{array}$ & $\begin{array}{l}4.1-6.4 \\
(5.2 \pm 0.664)\end{array}$ & $\begin{array}{l}54-106 \\
(87.6 \pm 16.855)\end{array}$ & $\begin{array}{l}127-656 \\
(319 \pm 169.045)\end{array}$ & $\begin{array}{l}199-572 \\
(335 \pm 118.93)\end{array}$ & $\begin{array}{l}0-0.1 \\
(0.03 \pm 0.03)\end{array}$ \\
\hline & Tap & 1 & 27.2 & 7.1 & 6.4 & 5.4 & 67 & 115 & 221 & 0 \\
\hline \multirow[t]{5}{*}{8} & Hand pump & 2 & $\begin{array}{l}27-27.6 \\
(27.3 \pm 0.3)\end{array}$ & $\begin{array}{l}6.3-6.6 \\
(6.4 \pm 0.15)\end{array}$ & $\begin{array}{l}5.6-6.8 \\
(6.2 \pm 0.6)\end{array}$ & $\begin{array}{l}5-6.4 \\
(5.7 \pm 0.7)\end{array}$ & $\begin{array}{l}90-120 \\
(105 \pm 15)\end{array}$ & $\begin{array}{l}421-1015 \\
(718 \pm 297)\end{array}$ & $\begin{array}{l}367-885 \\
(626 \pm 259)\end{array}$ & $\begin{array}{l}0-0.3 \\
(0.15 \pm 0.15)\end{array}$ \\
\hline & Well & 2 & $\begin{array}{l}28-28.2 \\
(28.1 \pm 0.1)\end{array}$ & $\begin{array}{l}6.3-6.5 \\
(6.4 \pm 0.1)\end{array}$ & $\begin{array}{l}6.8-7 \\
(6.9 \pm 0.05)\end{array}$ & $\begin{array}{l}6-8 \\
(7 \pm 1)\end{array}$ & $\begin{array}{l}40-102 \\
(71 \pm 31)\end{array}$ & $\begin{array}{l}608-1043 \\
(825.5 \pm 217.5)\end{array}$ & $\begin{array}{l}530-909 \\
(719.5 \pm 189.5)\end{array}$ & $\begin{array}{l}0-0.3 \\
(0.15 \pm 0.15)\end{array}$ \\
\hline & Tap & 1 & 25 & 6.8 & 3.8 & 5.2 & 48 & 574 & 500 & 0 \\
\hline & Pond & 1 & 30.2 & 6.8 & 2.6 & 2.6 & 122 & 425 & 370 & 0 \\
\hline & River & 1 & 31 & 7 & 3.1 & 3.1 & 76 & 280 & 244 & 0 \\
\hline \multirow[t]{4}{*}{9} & Hand pump & 6 & $\begin{array}{l}27-29 \\
(27.8 \pm 0.264)\end{array}$ & $\begin{array}{l}6.5-6.7 \\
(6.6 \pm 0.124)\end{array}$ & $\begin{array}{l}5-10 \\
(7.3 \pm 1.14)\end{array}$ & $\begin{array}{l}5.2-6.8 \\
(5.8 \pm 1.344)\end{array}$ & $\begin{array}{l}48-218 \\
(90.66 \pm 23.022)\end{array}$ & $\begin{array}{l}190-773 \\
(423.5 \pm 79.389)\end{array}$ & $\begin{array}{l}166-672 \\
(361.16 \pm 70.862)\end{array}$ & $\begin{array}{l}0-0.1 \\
(0.016 \pm 0.014)\end{array}$ \\
\hline & Well & 3 & $\begin{array}{l}24-25.4 \\
(24.8 \pm 0.416)\end{array}$ & $\begin{array}{l}6.5-6.8 \\
(6.6 \pm 0.1)\end{array}$ & $\begin{array}{l}5-8 \\
(6.9 \pm 1.06)\end{array}$ & $\begin{array}{l}5.4-8.8 \\
(7 \pm 0.986)\end{array}$ & $\begin{array}{l}34-44 \\
(38 \pm 3.055)\end{array}$ & $\begin{array}{l}212-429 \\
(305.33 \pm 64.452)\end{array}$ & $\begin{array}{l}184-373 \\
(290 \pm 55.758)\end{array}$ & 0 \\
\hline & Tap & 2 & $\begin{array}{l}27.4-30 \\
(28.7 \pm 1.3)\end{array}$ & $\begin{array}{l}6.7-6.8 \\
(6.7 \pm 0.05)\end{array}$ & $\begin{array}{l}5.6-7.4 \\
(6.5 \pm 0.9)\end{array}$ & $\begin{array}{l}5.6-7 \\
(6.3 \pm 0.7)\end{array}$ & $\begin{array}{l}40-84 \\
(62 \pm 22)\end{array}$ & $\begin{array}{l}226-1105 \\
(666.5 \pm 439.5)\end{array}$ & $\begin{array}{l}197-963 \\
(580 \pm 383)\end{array}$ & $\begin{array}{l}0-0.4 \\
(0.2 \pm 0.2)\end{array}$ \\
\hline & River & 1 & 31 & 6.8 & 2.9 & 3.2 & 76 & 1508 & 143 & 0.1 \\
\hline \multirow[t]{4}{*}{10} & Hand pump & 3 & $\begin{array}{l}27.4-28.2 \\
(27.8 \pm 0.24)\end{array}$ & $\begin{array}{l}6.8-7.1 \\
(6.9 \pm 0.08)\end{array}$ & $\begin{array}{l}5-6.4 \\
(5.5 \pm 0.437)\end{array}$ & $\begin{array}{l}5.2-6.2 \\
(5.5 \pm 0.317)\end{array}$ & $\begin{array}{l}75-104 \\
(87.33 \pm 8.647)\end{array}$ & $\begin{array}{l}250-367 \\
(291 \pm 38.039)\end{array}$ & $\begin{array}{l}254-327 \\
(301.66 \pm 23.849)\end{array}$ & 0 \\
\hline & Well & 1 & 27 & 6.5 & 6 & 5.8 & 56 & 189 & 278 & 0 \\
\hline & Tap & 1 & 30 & 6.9 & 7.1 & 5.6 & 77 & 105 & 289 & 0.1 \\
\hline & ponds & 3 & $\begin{array}{l}30-31 \\
(30.4 \pm 0.29)\end{array}$ & $\begin{array}{l}6.8-7.7 \\
(7.1 \pm 0.284)\end{array}$ & $\begin{array}{l}2.9-3.4 \\
(3.1 \pm 0.145)\end{array}$ & $\begin{array}{l}2.9-3.6 \\
(3.33 \pm 0.218)\end{array}$ & $\begin{array}{l}70-82 \\
(76.33 \pm 3.48)\end{array}$ & $\begin{array}{l}105-143 \\
(122.33 \pm 11.095)\end{array}$ & $\begin{array}{l}250-310 \\
(283 \pm 17.578)\end{array}$ & $\begin{array}{l}0-.1 \\
(0.03 \pm 0.033)\end{array}$ \\
\hline \multirow[t]{3}{*}{11} & Hand pump & 2 & $\begin{array}{l}26-27 \\
(26.5 \pm 0.5)\end{array}$ & $\begin{array}{l}6.8-7 \\
(6.9 \pm 0.1)\end{array}$ & $\begin{array}{l}5-6.1 \\
(5.5 \pm 0.55)\end{array}$ & $\begin{array}{l}5.1-6.4 \\
(5.7 \pm 0.65)\end{array}$ & $\begin{array}{l}123-130 \\
(126.5 \pm 3.5)\end{array}$ & $\begin{array}{l}331-471 \\
(401 \pm 70)\end{array}$ & $\begin{array}{l}178-184 \\
(181 \pm 3)\end{array}$ & 0 \\
\hline & Well & 2 & $\begin{array}{l}25.4-27 \\
(26.2)\end{array}$ & $\begin{array}{l}7.2-7.4 \\
(7.3 \pm 0.1)\end{array}$ & $\begin{array}{l}6-7.2 \\
(6.6 \pm 0.6)\end{array}$ & $\begin{array}{l}4.6-5.4 \\
(5 \pm 0.4)\end{array}$ & $\begin{array}{l}138-140 \\
(139 \pm 1)\end{array}$ & $\begin{array}{l}212-941 \\
(576.5 \pm 364.5)\end{array}$ & $\begin{array}{l}820-1846 \\
(1333 \pm 5.3)\end{array}$ & $\begin{array}{l}0.2-0.9 \\
(0.55 \pm 0.35)\end{array}$ \\
\hline & Tap & 1 & 29 & 6.9 & 8 & 5.4 & 140 & 660 & 575 & 0 \\
\hline \multirow[t]{3}{*}{12} & Hand pump & 4 & $\begin{array}{l}27.4-29 \\
(28.1 \pm 0.33)\end{array}$ & $\begin{array}{l}6.5-7.1 \\
(6.8 \pm 0.177)\end{array}$ & $\begin{array}{l}5-8.2 \\
(6.1 \pm 0.732)\end{array}$ & $\begin{array}{l}5.1-6.2 \\
(5.7 \pm 0.242)\end{array}$ & $\begin{array}{l}73-100 \\
(86.75 \pm 5.878)\end{array}$ & $\begin{array}{l}124-301 \\
(213.5 \pm 42.868)\end{array}$ & $\begin{array}{l}198-315 \\
(243.5 \pm 26.66)\end{array}$ & 0 \\
\hline & Well & 3 & $\begin{array}{l}24.8-26.4 \\
(25.6 \pm 0.46)\end{array}$ & $\begin{array}{l}6.5-7.2 \\
(6.8 \pm 0.272)\end{array}$ & $\begin{array}{l}5.8-7 \\
(6.13 \pm 0.437)\end{array}$ & $\begin{array}{l}4-5.2 \\
(4.73 \pm 0.371)\end{array}$ & $\begin{array}{l}30.8-90 \\
(58.26 \pm 14.48)\end{array}$ & $\begin{array}{l}269-453 \\
(350.33 \pm 54.176)\end{array}$ & $\begin{array}{l}129-273 \\
(205.33 \pm 41.794)\end{array}$ & 0 \\
\hline & Tap & 3 & $\begin{array}{l}29.2-31 \\
(30.7 \pm 0.819)\end{array}$ & $\begin{array}{l}7.1-7.2 \\
(7.1 \pm 0.033)\end{array}$ & $\begin{array}{l}6.8-8.4 \\
(7.4 \pm 0.50)\end{array}$ & $\begin{array}{l}5.2-6 \\
(5.66 \pm 0.24)\end{array}$ & $\begin{array}{l}78-94 \\
(84 \pm 5.033)\end{array}$ & $\begin{array}{l}149-281 \\
(197.33 \pm 42.001)\end{array}$ & $\begin{array}{l}197-233 \\
(214.66 \pm 10.397)\end{array}$ & 0 \\
\hline
\end{tabular}

undesirable consequence on aquatic life such as leading to production of ammonia and hydrogen sulphide which causes negative effect on fish [33].

Alkalinity: The overall range of alkalinity of water samples examined was from 22 to $256 \mathrm{ppm}$ and the mean values ranged from $35 \pm 1$ to $144.75 \pm 40.359 \mathrm{ppm}$ (Table-1). It was observed that 14 samples were above $120 \mathrm{ppm}$ as prescribed by [34] guidelines. Similar findings were observed by [35] who recorded alkalinity upto $290 \mathrm{mg} / \mathrm{l}$ in the river water. The value of alkalinity may be due to high temperature in summer and increased level of bicarbonate because of high rate of photosynthesis [36]. These findings are not in line with the observation of $[37,38]$ who recorded lower alkalinity of water samples ranging from $20-110 \mathrm{mg} / \mathrm{l}$ and 110 to $149 \mathrm{mg} / \mathrm{l}$ respectively.

However it does agree with the findings of [39, 40] who recorded higher alkalinity varying from 170$870 \mathrm{mg} / \mathrm{l}$ and $210-910 \mathrm{mg} / \mathrm{l}$, respectively.

The variation in alkalinity of water is due to the presence of bicarbonate, carbonate and hydroxide compound of calcium, sodium and potassium.

Conductivity: Conductivity of water samples varied from 76 to $1636 \mu \mathrm{S} / \mathrm{cm}$ and the mean values range from $83 \pm 1.15$ to $1508 \pm 0 \mu \mathrm{S} / \mathrm{cm}$ (Table-1). It was noticed that 2 samples were above the prescribed level of 1400 
Table-2. Mean Concentration (ppm) of Lead and Cadium in water samples (Mean \pm SEM)

\begin{tabular}{llcc}
\hline S.No. & Sources & Lead $(\mathbf{P b})$ ppm Mean \pm SE & Cadmium (Cd) ppm Mean \pm SE \\
\hline 1 & Wells $(\mathrm{n}=27)$ & $0.07 \pm 0.03$ & $0.03 \pm 0.01$ \\
2 & Hand pump (44) & $0.08 \pm 0.02$ & $0.01 \pm 0.00$ \\
3 & Taps (20) & $0.11 \pm 0.03$ & $0.01 \pm 0.00$ \\
4 & Ponds (6) & $0.04 \pm 0.01$ & $0.02 \pm 0.01$ \\
5 & Rivers (3) & $0.05 \pm 0.04$ & $0.07 \pm 0.02$ \\
\hline
\end{tabular}

$\mu \mathrm{S} / \mathrm{cm}$ by [41]. The results were in partial accordance with those of $[42,43]$ who found the electrical conductivity of water was varying from 334-1640 $\mu \mathrm{S} / \mathrm{cm}$ and 423-1197 $\mu \mathrm{S} / \mathrm{cm}$, respectively. These findings are not in conformity with the observation of [44] who recorded lower conductivity of water samples ranging between $468-810 \mu \mathrm{S} / \mathrm{cm}$.

However, it does not agree with the findings of [45-47] who recorded higher electrical conductivity ranging from $755-1898 \mu / \mathrm{cm}, 386-2827 \mu / \mathrm{cm}$ and $400-3500 \mu \mathrm{S} / \mathrm{cm}$, respectively. The high electrical conductivity value might be due to the presence of high amount of dissolved inorganic substances in ionized form.

Total dissolved solids: The estimate of total dissolved solid of samples ranged from 67 to $1846 \mathrm{mg} / \mathrm{l}$. The mean values ranged from $73 \pm 1$ to $1422 \pm 0 \mathrm{mg} / \mathrm{l}$ (Table1). It was observed that 4 samples showed above the permissible level of $1000 \mathrm{mg} / \mathrm{l}$ prescribed by [48]. These findings are not in line with [49] who reported the TDS values ranged from 145 to $245 \mathrm{mg} / \mathrm{l}$. Higher TDS was reported in well $(1846 \mathrm{mg} / \mathrm{l})$. However the presence of high levels of TDS in water may be objectionable to consumers owing to the resulting taste and to excessive scaling in water pipes, heaters, boilers, and household appliances. Some dissolved organic matter may contribute to increased level of TDS which also indicates that water is polluted [50].

While, water with extremely low concentrations of TDS may also be unacceptable to consumers because of its flat and insipid taste.

In a similar study conducted by [51] the TDS of water samples were found to be in higher range from 666.7 to $3666.6 \mathrm{mg} / \mathrm{l}$, before treatment but within a similar range 509.2 to $1472.3 \mathrm{mg} / \mathrm{l}$ after treatment. The results were in partial accordance with those of [52] who recorded the TDS ranging from $240-1650 \mathrm{mg} / \mathrm{l}$.

High TDS might be due to the presence of large number of organic salts as carbonate, bicarbonate, sodium, potassium and calcium and also some nonvolatile substance which are solid at room temperature.

Salinity: The overall range of salinity in water was from 0 to $0.9 \mathrm{ppt}$ and the mean values ranged from $0.016 \pm 0.014$ to $0.7 \pm 0 \mathrm{ppt}$ (Table- 1 ). These findings are supported by [53] who conducted analyses of tube well water and found salinity of water ranging from 0.8 to $1 \mathrm{ppt}$.

These findings are not in line with [54] who recorded lower salinity in water ranging from 0.005 to $0.599 \mathrm{ppt}$.

In a similar study conducted by $[55,56]$ who reported higher salinity in the water samples ranging from 5 to $17 \mathrm{ppt}$ and 33 to $35 \mathrm{ppt}$, respectively.

The physico-chemical characteristic of river water in the study suggested that river and pond water is not suitable for drinking purpose.

Heavy metal estimation: In the present study, the concentrations of lead and cadmium were found more than the prescribed permissible limits of WHO [57]. The increase in the lead level may indicate presence of old pipes and industrial pollution [58], effect of combustion of petrol [59] and gasoline [60]. The increased levels may cause damage to brain, kidney if taken in high concentration [61]. The increase in level of cadmium indicates the pollution of water bodies by industrial activities [62], excessive uses of steel plating, nickel cadmium batteries. Exposure to cadmium causes kidney damage [63]. The accumulation of these heavy metals might be due to anthropogenic activities and important in public health point of view.

\section{Conclusion}

The gross appearance, odour and taste were agreeable except pond and river water which revealed high turbidity and foul smelling odour. Parameters like temp, $\mathrm{pH}, \mathrm{DO}, \mathrm{BOD}$, alkalinity, Conductivity, total dissolved solid and salinity was within the permissible limits of WHO. The lead and cadmium levels were slightly more than the permissible limits of WHO in all water samples, which could be important in public point of view. The physico-chemical characteristics in the study suggested that river and pond water is not suitable for drinking purpose. Regular estimation of the above mentioned parameters would be helpful to improve water quality.

\section{Authors' contribution}

All authors contributed equally. All author read and approved the final manuscript.

\section{Acknowledgements}

Authors are thankful to The Vice-Chancellor for providing the fund for this study and Dean, Ranchi Veterinary College for supporting the research.

\section{Competing interests}

Authors declare that they have no competing interest.

\section{References}

1. Lamikaran, A. (1999) Essential Microbiology for students and Practitioners of Pharmacy, Medicine and Microbiology, $2^{\text {nd }}$ Edn., Amkrabooks., p- 406.

2. Nikoladze, G.D.M. and Akastal, S. (1989) Water treatment for public and industrial supply. MIR Publisher Moscoul., p. 163 . 
3. Lemo, O.O. (2002) Bacteriology determination of water with long term storage (BSc. Thesis) UNAAB Abeokuta., p. 44.

4. Haloi, N. and Sarma, H.P. (2011) Ground Water Quality Assessment of some parts of Brahmaputra Flood plain in Barpeta district, Assam with special focus on Fluoride, Nitrate, Sulphate and Iron analysis, Int. J. Chem. Tech., 3(3): 1302-1308.

5. WHO, (1998): Guidelines for drinking water quality., $3^{\text {rd }}$ edn. World Health Organisation, Geneva.

6. Reza, R. and Singh. G. (2009) Physico- Chemical Analysis of Ground Water in Angul-Talcher Region of Orissa, India, Marsland Press, J.Am.Sci., 5(5):53-58.

7. Rao, S. M. and Mamatha, P. (2004) Water quality in sustainable water management. Cur. sci., 87 (7): 942-947.

8. Altman, S.J. and Parizek, R.R. (1995) Dilution of nonpoint source nitrate in ground water. J. Environ. Qual., 24: 707717.

9. Adekunle, A.S. (2009) Effects of Industrial Effluent on Quality of Well Water Within Asa Dam Industrial Estate, Ilorin, Nigeria., Nat. Sci., 7(1):34-39.

10. Ramakrishnaiah, C.R. (2009) Assessment of Water Quality Index for the Groundwater in Tumkur Taluk, Karnataka State, India. E-J. Chem., 6(2): 523-530.

11. Kumar, A., Bagavathiraj B. and Kumarji, B. (1996) Physicochemical and microbiological aspects of Courtallam water. Poll. Res., 15(2): 159-161.

12. Dahiya, S. and Kaur, A. (1999) Assessment of physicochemical characteristics of underground water in rural areas of tasham subdivisions, Bhiwani district, Haryana. Enviro. J. Poll., 6(4): 281-288.

13. WWF. (1998) Living planet report: over consumption in driving the rapid decline of the world's environments. World Wide Fund for Nature, Gland, Switzerland.

14. Oluyemi, E.A., Adekunlel A.S., Adenuga A.A. and Makinde W.O. (2010) Physico-chemical properties and heavy metal content of water sources in Ife North Local Government Area of Osun State, Nigeria. Afr. J. Environ. Sci. Technol., 4(10): 691-697.

15. Lamikaran, A. (1999) Essential Microbiology for students and Practitioners of Pharmacy, Medicine and Microbiology, $2^{\text {nd }}$ Edn. , Amkra books., p- 406.

16. Shittu, O.B., Olaitan, J.O. and Amusa, T.S. (2008) PhysicoChemical and Bacteriological Analyses of Water Used for Drinking and Swimming Purposes in Abeokuta, Nigeria. Afr. J. Biomed. Res., 11:285 -290.

17. Singh, S. and Mosley, L.M. (2003) Trace metal levels in drinking water on Viti Levu, Fiji Islands. S. Pac. J. Nat. Sci., 21:31-34.

18. Winkler, L.W. (1888) The determination of dissolved oxygen in water. Berline Deutsch Chan Gesellsch., 21: 2843.

19. APHA. (1971) Standard method for the examination of water and wastewater. Washington D.C., p 874.

20. APHA. (1975) Standard methods for the examination of water and waste water. $19^{\text {th }}$ Edn., American Public Health Association.

21. Tandon, H.L.S. (1999) Methods of analysis of soil, plants, waters and fertilizers. Fertilizer development and consultation organization. New Delhi., Pp: 1-144.

22. Mishra, A. and Bhatt, V. (2008) Physico-chemical and microbiological analysis of underground water in V.V. Nagar and nearby places of Anand district, Gujarat, India. E-J. Chem., 5(3): 487-492.

23. Khan, R.M., Jadhav, M.J. and Ustad, I.R. (2012) Physicochemical analysis of Triveni lake water of Amravati District in (MS) India. Biosci. Discovery., 3(1):64-66.

24. Bernard, E. and Ayeni, N. (2012) Physico-chemical analysis of groundwater samples of Bichi local government area of Kano state of Nigeria. ARPNJ. Sci. Technol., 2: 2225-7212.

25. Shittu, O.B., Olaitan, J.O. and Amusa, T.S. (2008) PhysicoChemical and Bacteriological Analyses of Water Used for Drinking and Swimming Purposes in Abeokuta, Nigeria. Afr. J. Biomed. Res., 11:285-290.
26. Pandey, S. K. and Tiwari, S. (2009) Physico-chemical analysis of ground water of selected area of Ghazipur city-A case study. Nat. Sci.., 7(1):17-20.

27. Simpi, B., Hiremath, S.M., Murthy, K.N.S., Chandrashekarappa, K.N., Patel, A. and Puttiah, E.T. (2011) Analysis of Water Quality Using Physico-Chemical Parameters Hosahalli Tank in Shimoga District, Karnataka, India. Global J. Sci. Front. Res., 11(3):31-34.

28. Budget, U.S. and Verma, A.K. (1985) Limnological studies on J.N.U. Lake, New Delhi, India. Bull. Bot. Soc. Sugar., 32: $16-23$.

29. WHO. (1996) Guidelines for drinking water quality, Geneva, $2^{\text {nd }} \mathrm{Edn}$.

30. ISI: 10500. (1991) Indian standards of drinking water specification, Bureau of Indian standard (BIS), New Delhi, India.

31. Usharani, K., Umarani, K., Ayyasamy, P. M., Shanthi, K. and Lakshmanaperumalsamy, P. (2010) Physico-chemical and bacteriological characteristics of Noyyal river and ground water quality of Perur, India. J. Appl. Sci. Environ. Manage., 14(2):29-35.

32. Kurup, R., Persaud, R., Caesar, J. and Raja, V. (2011) Microbiological and physiochemical analysis of drinking water in Georgetown, Guyana. Nat. Sci., 8(8): 261-265.

33. Boyd, C. E. and Lichikoppler, F. (1979) Water quality in fish pond culture research and development. No. 22 International Center for Agriculture. Experimental Station.Aubm.

34. WHO. (1973) Guidelines for drinking water quality. $3^{\text {rd }}$ Edn.World Health Organisation, Geneva, Washington, D.C.

35. Belorkar, S.A. (2010) Assessment of the deterioration in physio-chemical and microbiological quality of Shivnath River Water in Durg District, India. E-J. Chem., 7(3): 733738.

36. Hujare, M. S. (2008) Seasonal variation of physico-chemical parameters in the perennial tank of Talsande, Maharashtra. Ecotoxicol. Environ. Monitor., 18(3): 233-242.

37. Odeyemi, A.T., Dada, A.C., Ogunbanjo, O.R. and Ojo, M.A. (2010) Bacteriological, physicochemical and mineral studies on Awedele spring water and soil samples in Ado Ekiti, Nigeria. Afr. J. Environ. Sci. Technol., 4(6):319-327.

38. Pandey, S. K. and Tiwari, S. (2009) Physico-chemical analysis of ground water of selected area of Ghazipur city-A case study. Nat. Scie., 7(1):17-20.

39. Patil, V.T. and Patil, P.R. (2010) Physico-chemical analysis of selected ground water samples of Amalner Town in Jalgaun District, Maharastra, India. E- J. Chem., 7(1): 111116.

40. Krishnan, R. R., Dharmaraj, K. and Kumari, B.D.R. (2007) A comparative study on the physicochemical and bacterial analysis of drinking, borewell and sewage water in the three different places of Sivakasi. J. Env. Biol., 28(1): 105-108.

41. WHO. (1973) Guidelines for drinking water quality. $3^{\text {rd }}$ Edn.World Health Organisation, Geneva, Washington, D.C.

42. Devi, S. and Premkumar, K. (2012) Physico-chemical analysis of groundwater samples near industrial area, Cuddalore District, Tamil Nadu, India. Int. J. Chem Tech Res., 4(1):29-34.

43. Hacioglu, N. and Dulger, B. (2009) Monthly variation of some physico-chemical and micribiological parameters in Big Stream (Biga, Canakkale, Turkey). Afr. J. of Biot.8(9): 1929-1937.

44. Shittu, O.B., Olaitan, J.O. and Amusa, T.S. (2008) PhysicoChemical and Bacteriological Analyses of Water Used for Drinking and Swimming Purposes in Abeokuta, Nigeria. Afr. J. Biomed. Res., 11: 285 -290.

45. Rao, V.S., Prasanthi, S., Shanmukha, K.J.V. and Prasad, K.R.S. (2012) Physico- chemical analysis of water samples of Najendle area in Gunter District of Andhra Pradesh, India. Inter. J. Chem. Tech. Res., 4(2): 691-699.

46. Patil, V.T. and Patil, P.R. (2010) Physico-chemical analysis of selected ground water samples of Amalner Town in Jalgaun District, Maharastra, India. E- J. Chem., 7(1): 111- 
116.

48. WHO. (1973) Guidelines for drinking water quality. $3^{\text {rd }}$ Edn. World Health Organization, Geneva, Washington, D.C.

49. Pandey, S. K. and Tiwari, S. (2009) Physico-chemical analysis of ground water of selected area of Ghazipur city-A case study. Nat. Sci., 7(1):17-20.

50. Rao, V.S., Prasanthi, S., Shanmukha, K.J.V. and Prasad, K.R.S. (2012) Physico-chemical analysis of water samples of Najendle area in Gunter District of Andhra Pradesh, India. Inter J. Chem. Tech. Res., 4(2): 691-699.

51. Farah, N., Zia, M.A., Rehman, K.U. and Sheikh, M.A. (2002) Quality characteristics and treatment of drinking water of Faisalabad City., Int. J. Agri. Biol., 4(3): 347-349.

52. Suresh, T. and Kottureshwara, N.M. (2009) Assessment of groundwater quality of borewells water of Hospettaluka region in Bellary district, Karnataka, India. Ras. J. Chem., 2(1): 221-233.

53. Islam, M.S., Siddika, A., Khan, M.N.H., Goldar, M.M., Sadique, M.A., Kabir, A.N.M.H.,Huq, A. and Colwell, R.R. (2001) Microbiological analysis of tube-well water in a rural area of Bangladesh. App. Env. Micro., 67 (7): 3328-3330.

54. Obire, O., Aguda, M. and Putheti, R.R (2009) Impact of human activities on drinking water quality. J. Bas. App. Biol., 2(3\& 4): 52-58.

55. Abowei, J.F.N (2010) Salinity, dissolved oxygen, $\mathrm{pH}$ and surface Water temperature conditions in Nkoro River, Niger Delta, Nigeria. Adv. J. Food. Sci. Techno., 2(1): 36-40.

56. Sugumar, G., Chrisolite, B., Velayutham, P., Selvan, A., and Ramesh, U. (2008) Occurrence and seasonal variation of bacterial indicators of faecal pollution along Thoothukuti coast, Tamil Nadu. J. Environ. Biol., 29(3): 387-391.

57. WHO. (2003) International year of fresh water. General assembly resolution A/RES/55/196. Official website: www. wateryear2003.org., Retrieved on 01-10-2012.

58. Gebrekidan, M. and Samuel, Z. (2011) Concentration of Heavy Metals in Drinking Water from Urban Areas of the Tigray Region, Northern Ethiopia., M. E. J. S., 3(1): 105121.

59. Hardman, D.J., Mceldowney, S. and Watte, S. (1999) Pollution, ecology and biotreatment. Longman Scientific, Technical, England.

60. Banat, I.M., Hassan, E.S., El-Shahawi, M.S. and Abu-Hilal, A.H. (1998) Postgulf- war assessment of nutrients, heavy metal ions, hydrocarbons, and bacterial pollution levels in the United Arab Emirates coastal waters. Environ. Inter., 24(2): 109-116.

61. Hanaa, M., Eweida, A and Farag, A. (2000) Heavy metals in drinking water and their environmental impact on human health. International Conference on Environmental Hazards Mitigation, Cairo University, Egypt., pp. 542-556.

62. Nassef, M., Hannigan, R. EL., Sayed, K.A and Tahawy, M.S.El. (2006) Determination of some heavy metals in the environment of Sadat industrial city. Proceeding of the 2nd Environmental Physics Conference, Cairo University, Egypt., pp. 145-152.

63. Rajappa, B., Manjappa, S. and Puttaiah, E.T. (2010) Monitoring of heavy metal concentration in groundwater of Hakinaka Taluk, India., Contemp. Eng. Sci..,3(4):183-190. 